Research Paper

\title{
Downregulated pseudogene CTNNAP1 promote tumor growth in human cancer by downregulating its cognate gene CTNNA1 expression
}

\author{
Xiangjian Chen ${ }^{1,2}$, Hua Zhu ${ }^{3}$, Xiaoli Wu ${ }^{4}$, Xuemeng Xie ${ }^{2}$, Guanli Huang ${ }^{5}$, Xiaoqun \\ $\mathbf{X u}^{6}{ }^{6}$, Shi Li ${ }^{7}$, Chungen Xing ${ }^{1}$ \\ ${ }^{1}$ Department of General Surgery, The Second Affiliated Hospital of Soochow University, Suzhou, Jiangsu, P.R. China \\ ${ }^{2}$ Department of General Surgery, The First Affiliated Hospital of Wenzhou Medical University, Wenzhou, Zhejiang, P.R. China \\ ${ }^{3}$ Department of Obstetrics and Gynecology, The First Affiliated Hospital of Wenzhou Medical University, Wenzhou, Zhejiang, \\ P.R. China \\ ${ }^{4}$ Department of Gastroenterology, The First Affiliated Hospital of Wenzhou Medical University, Wenzhou, Zhejiang, P.R. China \\ ${ }^{5}$ Department of Surgical Oncology, The First Affiliated Hospital of Wenzhou Medical University, Wenzhou, Zhejiang, P.R. China \\ ${ }^{6}$ Operating Room, The First Affiliated Hospital of Wenzhou Medical University, Wenzhou, Zhejiang, P.R. China \\ ${ }^{7}$ Department of Urology, The First Affiliated Hospital of Wenzhou Medical University, Wenzhou, Zhejiang, P.R. China \\ Correspondence to: Chungen Xing, email: xingcg@qq.com \\ Xiangjian Chen, email: wz1370666@126.com \\ Keywords: CRC, ceRNA, qRT-PCR, InCRNA, CCK-8
}

Received: March 27, $2016 \quad$ Accepted: June 17, $2016 \quad$ Published: July 25, 2016

\section{ABSTRACT}

Accumulating evidence indicates that deregulation of cancer-associated pseudogene is involved in the pathogenesis of cancer. In the study, we demonstrated that pseudogene CTNNAP1, for the CTNNA1 gene, was dysregulated in colorectal cancer and the degree of dysregulation was remarkably associated with tumor node metastasis (TNM) stage $(P<0.05)$. The mechanistic experiments revealed that pseudogene CTNNAP1 played a pivotal role in the regulation of its cognate gene CTNNA1 by competition for microRNA-141. Moreover, gain-of-function approaches showed that overexpression of CTNNAP1 or CTNNA1 significantly inhibited cell proliferation and tumor growth in vitro and in vivo by inducing G0/G1 cell cycle arrest. Our findings add a new regulatory circuit via competing endogenous RNA (ceRNA) cross-talk between pseudogene CTNNAP1 and its cognate gene CTNNA1, and provide new insights into potential diagnostic biomarker for monitoring human colorectal cancer.

\section{INTRODUCTION}

Colorectal cancer $(\mathrm{CRC})$ is the third most commonly diagnosed malignancy and the leading cause of cancer-related deaths in the world [1]. In fact, throughout the last few decades, epidemiological studies has shown that multiple environmental factors, genetic or epigenetic abnormalities are involved in the initiation ad progression of CRC $[2,3]$. Despite recent advances in diagnostic techniques and medical treatment, the overall survival of CRC patients remains still relatively low. Therefore, it is urgently needed to investigate the detailed pathophysiological mechanisms contributing to CRC which provide fundamental information for early diagnosis and treatment of CRC.
Lately, advances in the analysis of whole-genome sequencing data have showed that most genomic sequences is transcribed as non-coding RNA species, including long non-coding RNA (lncRNAs), pseudogenes and microRNAs etc. Numerous studies demonstrate that these non-coding transcripts are implicated in regulation of various cellular processes [4-7]. In recent studies, pseudogenes, which were recognized as a new class of non-coding RNAs, have been discovered sharing similar nucleotide sequence with their parental protein-coding genes [8]. However, these special genes lost their ability to produce functional protein products mostly arising as a consequence of premature stop codons or disabling mutations [9]. Similar to other non-coding RNAs, pseudogenes have been discovered to exert important roles 
in a variety of biological processes and human diseases, particularly in tumorigenesis [10-13]. To date, increasing evidence indicates that pseudogenes can act as competing endogenous RNA (ceRNA) to sustain the expression of their parental genes by competing for the binding of some of the same microRNA molecules [14]. As an example, the first ceRNA PTENP1 sequesters microRNAs (microRNA-21, microRNA-19b and microRNA-20a) away from its mRNA target PTEN, thereby influencing parent gene expression [15]. In the recently reported study by Yang W and colleagues [16], Foxo3 pseudogene (Foxo3P) could suppress tumor growth and angiogenesis by functioning as a sponge for microRNAs, and upregulate expression of the forkhead family of transcription factors, Foxo3.

In the present study, we investigated that pseudogene CTNNAP1 was aberrantly expressed in CRC and was positively associated with CTNNA1 expression. Furthermore, gain-of-function assays were further explored that pseudogene CTNNAP1 could act as a ceRNA to increase CTNNA1 gene expression through competition for microRNA-141, subsequently inhibiting cell proliferation and tumor growth. This study showed the first evidence for the cross-talk between CTNNAP1 and CTNNA1 via competing for microRNA-141, shedding a better understanding of molecular etiology of CRC.

\section{RESULTS}

\section{CTNNAP1 was downregulated in CRC}

As an intriguing class of lncRNAs, recent evidence increasingly discovered that pseudogenes have crucial roles in normal physiology as well as quite recently in the context of cancer. To evaluate the expression of pseudogene CTNNAP1, we performed qRT-PCR assay in a cohort of 56 pairs of CRC tissues and paired nontumor tissues. The result showed that the expression of CTNNAP1 was downregulated in $70 \%$ tumor samples $(39 / 56)$ compared to adjacent normal samples $(P<0.05$; Figure 1A). Additionally, CTNNAP1 subcellular localization was further analyzed in CRC cell lines. As showed in Figure 1B, CTNNAP1 was predominantly detectable in the cytoplasm (more than $75 \%$ ) than in the nucleus of fractionated SW480 and SW620 cells.

We then sought to determine the correlation of CTNNAP1 expression with clinicopathological features of CRC patients to assess its clinical significance. According to the median value $(0.68)$ of relative CTNNAP1 expression in CRC tissues, $56 \mathrm{CRC}$ patients were classified into high group $(\mathrm{n}=28, C T N N A P 1$ expression ratio $>0.68)$ and low group $(\mathrm{n}=28, C T N N A P 1$ expression ratio $<0.68$ ). We found that $C T N N A P 1$ expression levels in CRC tissues were remarkably associated with tumor node metastasis (TNM) staging $(P<0.05$; Table 1$)$. More importantly, CRC patients with advanced TNM stage (III and IV) exhibited decreased CTNNAP1 expression than those with low TNM stage (I and II) $(P<0.05$; Figure $1 \mathrm{C})$.

We further evaluated the cognate gene CTNNA1 of pseudogene CTNNAP1 expression in CRC clinical samples. CTNNA1 expression level is remarkably lower in CRC tissues in comparison with matched normal tissues (Figure 1D), and its expression is positively correlated with pseudogene CTNNAP1 expression level $(P<0.001$, $\mathrm{R}^{2}=0.399$ ) (Figure 1E). Taken together, these analyses indicated that CTNNAP1 may be a potential predictor for $\mathrm{CRC}$ development and progression.

\section{MicroRNA-141 inhibited pseudogene CTNNAP1 and its cognate gene CTNNA1 in CRC}

Pseudogenes are believed quite recently to play important roles in varies of diseases via competing for the binding of common microRNAs molecule with their parental genes, thereby liberating mRNA transcripts expression of microRNAs targets $[17,18]$. In addition, since the positive expression trend between pseudogene CTNNAP1 and its cognate gene CTNNA1, we further determined whether CTNNAP1 can regulate the expression of CTNNA1 through operating as a ceRNA. Based on the bioinformatics tools and the reference [11], 4 potential microRNAs binding sites scattered the CTNNAP1 transcript as well as the sequence of CTNNA1 3'-UTR (microRNA-141, microRNA-18b, microRNA-33a and microRNA-9). Among these microRNAs, microRNA-141 was found to be up-regulated in the same CRC tissues in comparison with matched normal tissues (Figure 2A). Notably, microRNA-141 had been reported to promote cell growth, cell cycle progression and tumor invasion in CRC [19]. In addition, correlation analyses revealed that microRNA-141 significantly correlated with the expression of CTNNAP1 and CTNNA1 in the CRC tissues $\left(P<0.001, \mathrm{R}^{2}=0.317\right.$ for $C T N N A P 1 ; P<0.001, \mathrm{R}^{2}=0.304$ for CTNNA1) (Figure 2B).

Considering the potential binding sites for microRNA-141 in CTNNAP1 and CTNNA1 genes (Supplementary Table S1) as well as the coordinated expression levels of CTNNAP1, CTNNA1 and microRNA-141, we performed dual luciferase reporter assays to investigate whether CTNNAP1 and CTNNA1 were regulated by microRNA-141. Reporter plasmids containing 3'-UTR of CTNNA1 (RLuc-CTNNA1-WT or RLuc-CTNNA1-MU) (Figure 2C), which contains wildtype or mutant microRNA-141 binding sites transfected with microRNAs mimics or negative controls into CRC cells. The result showed that luciferase activity from the RLuc-CTNNA1-WT were significantly reduced by $47 \%$ and $35 \%$ in SW480 and SW620 cells compared with the negative controls (Figure 2D and 2E). Furthermore, reporter plasmids containing the wild type 3'-UTR of CTNNA1 were subsequently transfected plasmid encoding CTNNAP1 (pcDNA3.1-CTNNAP1) or microRNA-141 
inhibitors along with microRNAs mimics. Expression of CTNNAP1 and knockdown of microRNA-141 partially abrogated the inhibitory effect of microRNA-141 (Figure $2 \mathrm{D}$ and $2 \mathrm{E}$ ). As we expected, luciferase activity of reporter plasmids containing the mutant CTNNA1 3'-UTR was not affected in cells which were transfected microRNA-141 mimics with inhibitors or plasmid encoding CTNNAP1 in comparison with controls (Figure 2D and 2E), suggesting a direct interactions between microRNA-141 and its putative recognition sites. Subsequent qRT-PCR analysis further showed that overexpression of microRNA-141 in SW480 and SW620 cells decreased the expression of CTNNA1 mRNA than the controls, whereas the inhibitory effect of microRNA-141 on CTNNA1 expression was completely abolished by the introduction of CTNNAP1 and knockdown of microRNA-141 (Figure 2F). Together, these data indicate that pseudogene CTNNAP1 can function as microRNA-141 decoy, thereby increasing its cognate gene CTNNA1 expression by sequestering microRNAs.

\section{The half-life of CTNNAP1 and CTNNA1 decreased by microRNA-141}

We measured the CTNNAP1 and CTNNA1 halflife after inhibiting transcription by incubating cells with actinomycin D using qRT-PCR. As showed in Figure 2G and $2 \mathrm{H}$, the transcript levels of the CTNNAP1 and CTNNA1
A
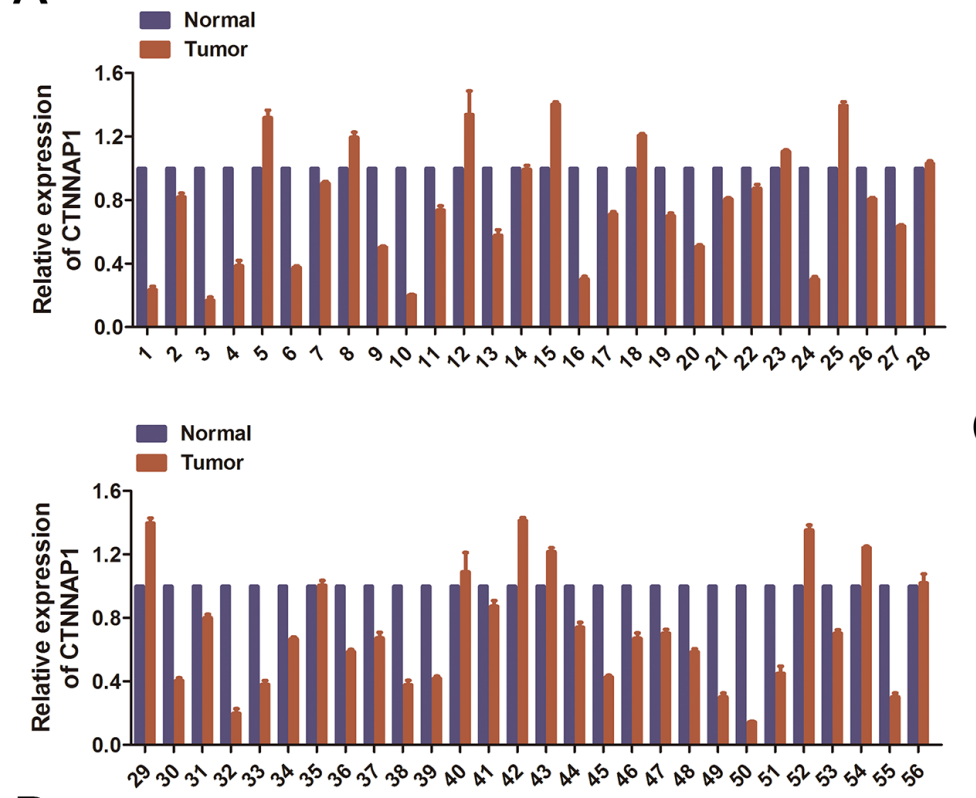

B

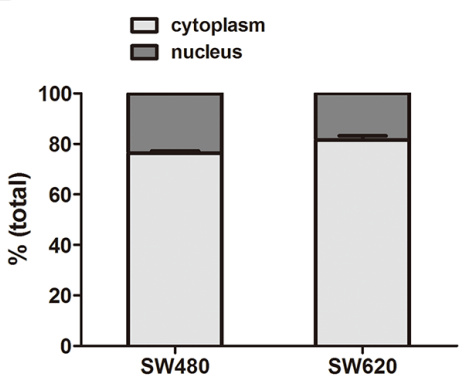

C

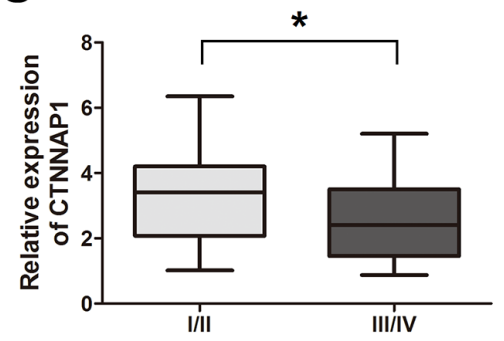

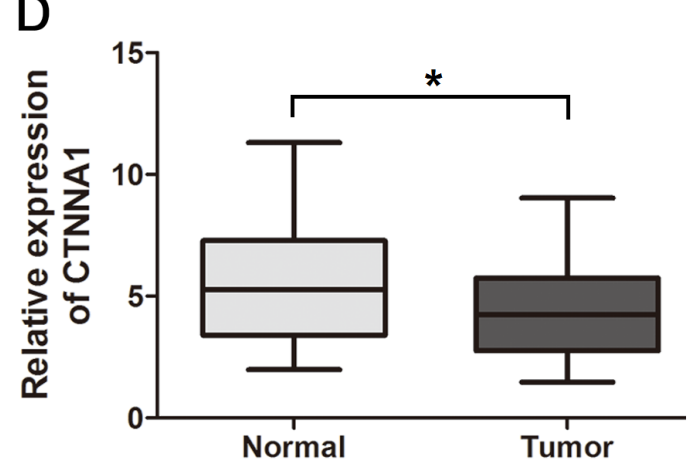

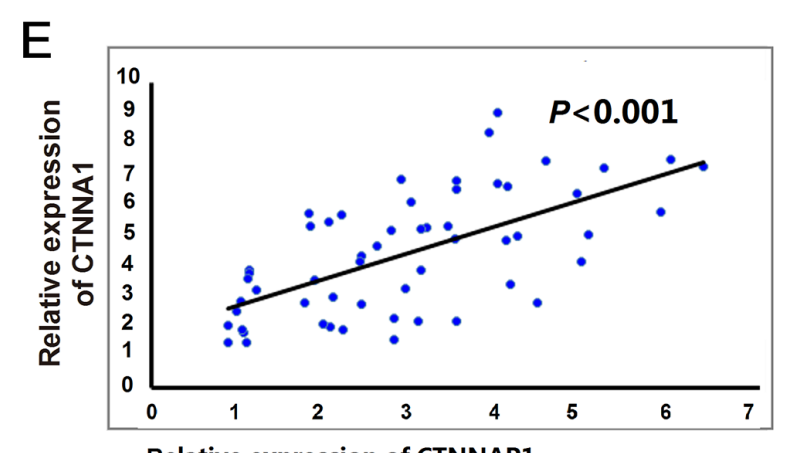

Relative expression of CTNNAP1

Figure 1: Expression levels of pseudogene CTNNAP1 and its cognate gene CTNNA1 in CRC. A. Relative expression of pseudogene CTNNAP1 in a cohort of 56 pairs of CRC tissues and paired nontumor tissues by real-time PCR. Data presented in tumor tissues is normalized to normal tissues. $P<0.001$, paired $t$-test. B. RNA of nuclear and cytoplasmic fractions in CRC cell line SW480 and SW620 was determined by qRT-PCR. CTNNAP1 was mostly located in the cytoplasmic fractions. Mean \pm S.D. are shown (n=3). C. CTNNAP1 expression was significantly downregulated in CRC patients with advanced TNM stage (III and IV) than those with low TNM stage (I and II). ${ }^{*} P<0.05$. D. $C T N N A 1$ is downregulated in CRC tissues compared with adjacent normal tissues $(P<0.05$, paired $t$-test). E. The expression of CTNNAP1 is positively correlated with CTNNA1 in CRC tissues $\left(P<0.001 ; \mathrm{R}^{2}=0.3987\right)$. The data were obtained by the logistic regression analysis.). 
Table 1: The correlation between $C N T T A 1$ and $C N T T A P 1$ expression and the clinical pathological factors of colorectal cancer patients

\begin{tabular}{|c|c|c|c|c|c|c|c|c|c|c|c|}
\hline \multirow{3}{*}{$\begin{array}{l}\text { Parameters } \\
\text { Gender }\end{array}$} & \multirow{2}{*}{$\begin{array}{l}\text { Total } \\
(\mathrm{N})\end{array}$} & \multicolumn{4}{|c|}{ Expression of CNTTA1 } & \multirow{2}{*}{$\boldsymbol{P}_{\text {value }}{ }^{*}$} & \multicolumn{4}{|c|}{ Expression of CNTTAP1 } & \multirow{2}{*}{$\boldsymbol{P}_{\text {value }}$} \\
\hline & & \multicolumn{2}{|c|}{ High } & \multicolumn{2}{|c|}{ Low } & & \multicolumn{2}{|c|}{ High } & \multicolumn{2}{|c|}{ Low } & \\
\hline & & & & & & & & & & & \\
\hline male & 28 & 15 & 26.79 & 13 & 23.21 & 0.592 & 12 & 21.43 & 16 & 28.57 & 0.285 \\
\hline female & 28 & 13 & 23.21 & 15 & 26.79 & & 16 & 28.57 & 12 & 21.43 & \\
\hline \multicolumn{12}{|l|}{ Age } \\
\hline$<50$ & 22 & 12 & 21.43 & 10 & 17.86 & 0.584 & 13 & 23.21 & 9 & 16.07 & 0.278 \\
\hline$\geq 50$ & 34 & 16 & 28.57 & 18 & 32.14 & & 15 & 26.79 & 19 & 33.93 & \\
\hline \multicolumn{12}{|c|}{ Differentiation } \\
\hline High & 16 & 11 & 19.64 & 5 & 8.93 & 0.706 & 10 & 17.86 & 6 & 10.71 & 0.707 \\
\hline Middle & 28 & 9 & 16.07 & 19 & 33.93 & & 11 & 19.64 & 17 & 30.36 & \\
\hline Low & 12 & 8 & 14.29 & 4 & 7.14 & & 7 & 12.5 & 5 & 8.93 & \\
\hline \multicolumn{12}{|l|}{$\begin{array}{l}\text { Lymph node } \\
\text { metastasis }\end{array}$} \\
\hline No & 30 & 18 & 32.14 & 12 & 21.43 & 0.109 & 17 & 30.36 & 13 & 23.21 & 0.284 \\
\hline Yes & 26 & 10 & 17.86 & 16 & 28.57 & & 11 & 19.64 & 15 & 26.79 & \\
\hline \multicolumn{12}{|l|}{ Tumor size } \\
\hline$<2 \mathrm{~cm}$ & 25 & 12 & 21.43 & 13 & 23.21 & 0.788 & 10 & 17.86 & 15 & 26.79 & 0.179 \\
\hline$\geq 2 \mathrm{~cm}$ & 31 & 16 & 28.57 & 15 & 26.79 & & 18 & 32.14 & 13 & 23.21 & \\
\hline \multicolumn{12}{|l|}{ TNM stages } \\
\hline $\mathrm{I}+\mathrm{II}$ & 29 & 20 & 35.71 & 9 & 16.07 & $\mathbf{0 . 0 0 3}^{*}$ & 19 & 33.93 & 10 & 17.86 & $0.016^{*}$ \\
\hline III+IV & 27 & 8 & 14.29 & 19 & 33.93 & & 9 & 16.07 & 18 & 32.14 & \\
\hline
\end{tabular}

was declined in CRC cells after RNA synthesis was blocked with Actinomycin D in the presence of microRNA-141. Furthermore, the half-life of CTNNAP1 and CTNNA1 regulated by microRNA-141 was shorter in CRC cells $\left(\mathrm{t}_{1 / 2}=2 \mathrm{~h}\right.$ for CTNNAPI and $\mathrm{t}_{1 / 2}=4 \mathrm{~h}$ for CTNNA1 in SW480 cells; $\mathrm{t}_{1 / 2}=3 \mathrm{~h}$ for CTNNAPI and $\mathrm{t}_{1 / 2}=2 \mathrm{~h}$ for CTNNA1 in SW620 cells) after actinomycin D treatment than in control cells $\left(\mathrm{t}_{1 / 2}=5 \mathrm{~h}\right.$ for CTNNAP1 and $\mathrm{t}_{1 / 2}=6 \mathrm{~h}$ for CTNNA1 in $\mathrm{SW} 480$ cells; $\mathrm{t}_{1 / 2}=4 \mathrm{~h}$ for $C T N N A P 1$ and $\mathrm{t}_{1 / 2}=5 \mathrm{~h}$ for $C T N N A 1$ in SW620 cells). These results indicate that microRNA-141 could suppress CTNNAP1 and its cognate gene CTNNA1.

\section{Effects of pseudogene CTNNAP1 and its cognate gene $C T N N A 1$ on cell proliferation in vitro}

Pseudogene CTNNAP1, for the human alpha E-catenin CTNNA1 gene, was originally characterized by fluorescence in situ hybridization [20]. Several studies have assessed the tumor suppressive role of CTNNA1 in various tumors [21-24]. However, no studies have been conducted on the effects of CTNNA1 gene on the progression of CRC. To elucidate the functions of CTNNAP1 and CTNNA1 in
$\mathrm{CRC}$, a series of functional assays were performed to investigate the roles of CTNNAP1 and CTNNA1 in cell proliferation and tumor growth in SW480 and SW620 cells. pcDNA3.1-CTNNAP1 or pcDNA3.1-CTNNA1 were transfected into SW480 and SW620 cells, respectively, and the transfection efficiency of CTNNAP1 and CTNNA1 overexpression were subsequently confirmed by qRTPCR analysis. After $48 \mathrm{~h}$ post-transfection, the RNA levels of CTNNAP1 and CTNNA1 revealed that CTNNAP1 expression was increased by 11 -fold and 13-fold in SW480 and SW620 cells than the empty vector pcDNA3.1, respectively (Figure 3A). Similar to CTNNAP1, relative level of CTNNA1 was significantly up-regulated by 8 -fold and 10-fold in SW480 and SW620 cells than the empty vector pcDNA3.1, respectively (Figure $3 \mathrm{~A}$ ).

Subsequently, we measured the effects of CTNNA1 or CTNNAP1 ectopic expression on cell proliferation. The CCK-8 assay showed that the increased expression of CTNNA1 in CRC cells inhibited proliferation compared with the controls at day 4 (Figure 3B and 3C). Moreover, upregulation of CTNNAP1 similarly decreased cell growth in SW480 and SW620 cells (Figure 3B and 3C). 
A
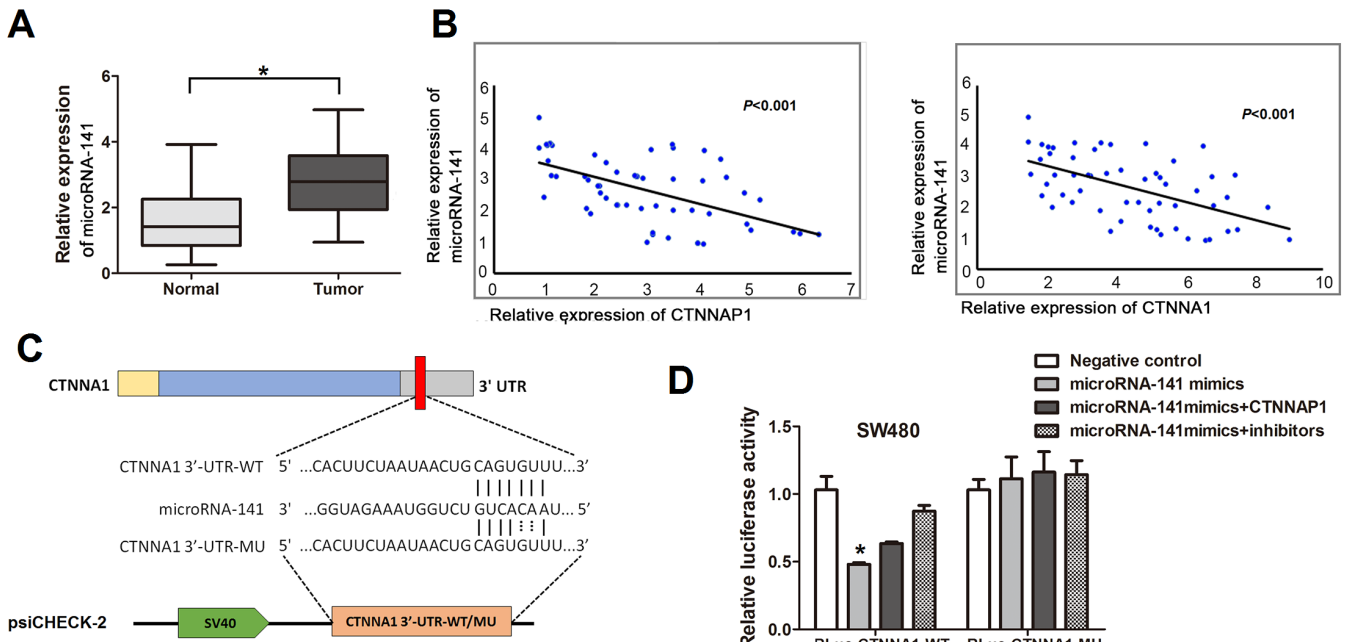

D

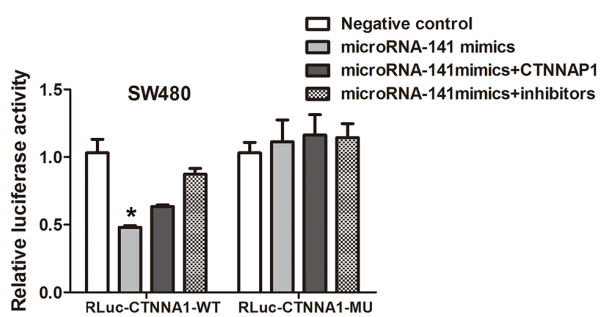

E

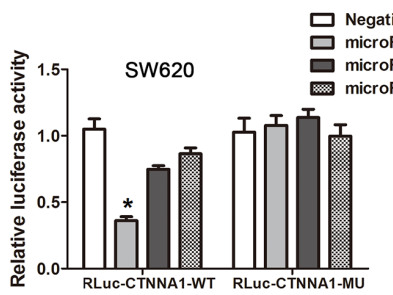

$\mathbf{F}$

G
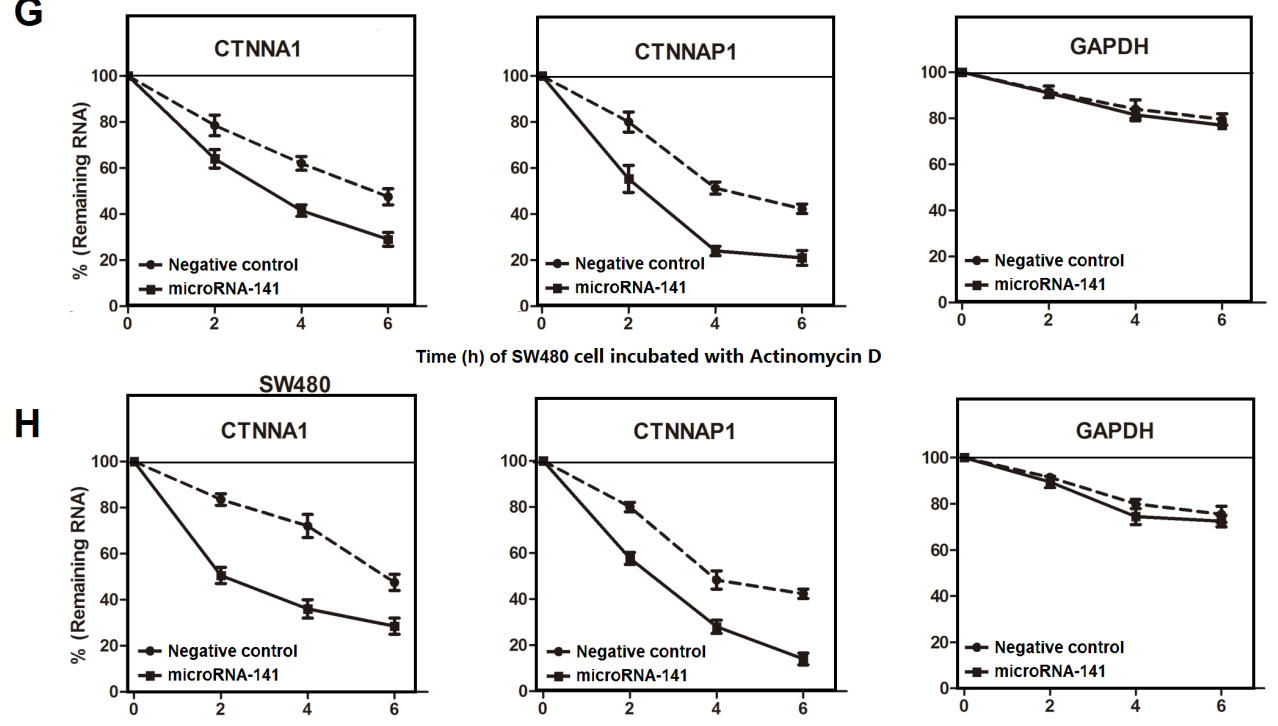

Time (h) of SW480 cell incubated with Actinomycin D
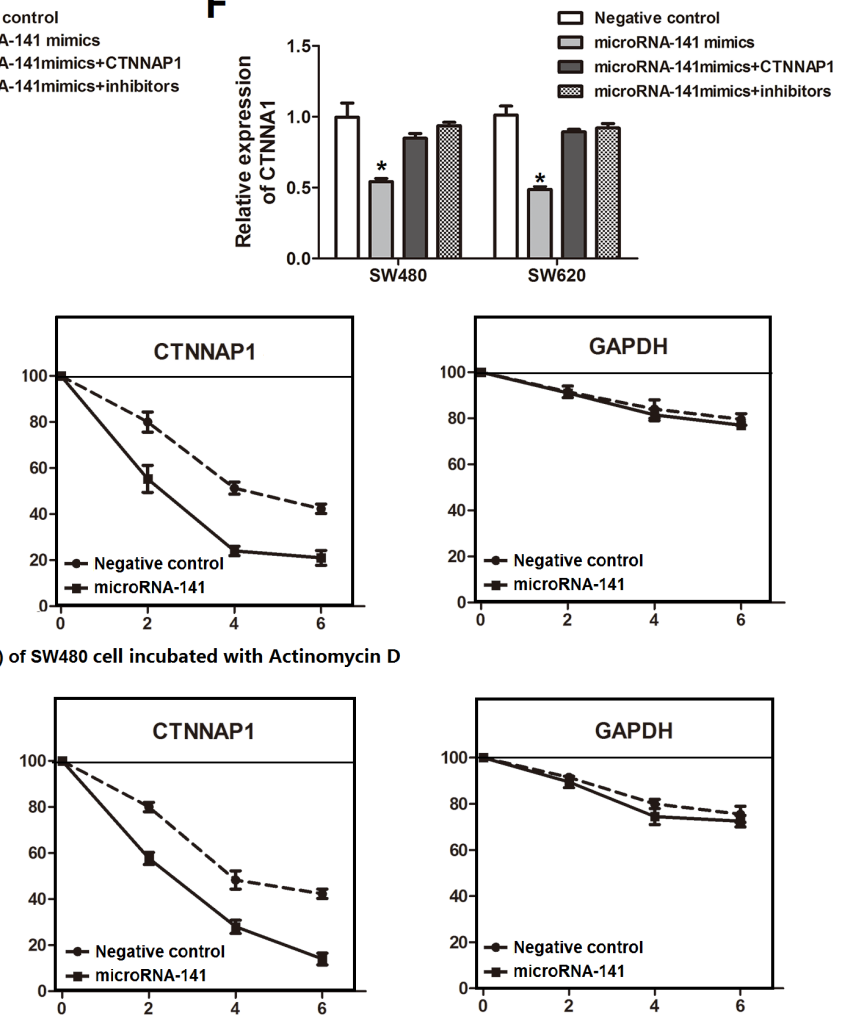

Time (h) of SW620 cell incubated with Actinomycin D

Figure 2: MicroRNA-141 inhibits the expression levels of pseudogene CTNNAP1 and its cognate gene CTNNA1 in CRC. A. The expression of microRNA-141 in CRC tissues and paired nontumor tissues. The expression of microRNA-141 is significantly upregulated in cancer tissues than normal controls. ${ }^{*} P<0.05$, paired $t$-test. B. Negative correlation between $C T N N A P 1, C T N N A 1$ expression and microRNA-141 level in tissues of 56 CRC patients $\left(P<0.001, \mathrm{R}^{2}=0.3166\right.$ between $C T N N A P 1$ and microRNA-141; $P<0.001, \mathrm{R}^{2}=0.3038$ between CTNNA1 and microRNA-141). The data were obtained using the logistic regression analysis. C. Schematic outlining of human CTNNA1 3'-UTR, and the position of the predicted microRNA-141 binding sites on CTNNA1 3'-UTR sequence. The wild-type CTNNA1 3'UTR containing the microRNA-141 recognition site (CTNNA1 3'-UTR-WT) or mutant CTNNA1 3'-UTR harboring mutated microRNA-141 binding site (CTNNA1 3'-UTR-MU) was cloned downstream of the luciferase gene. D. and E. Luciferase reporter containing the wild type CTNNA1 3'-UTR or mutant CTNNA1 3'-UTR were transfected plasmid harboring full length CTNNAP1 (pcDNA3.1-CTNNAP1) or microRNA-141 inhibitors in combination with microRNA-141 mimics. The luciferase activity was determined by luciferase reporter assays. Data are mean $\pm \mathrm{SD}(\mathrm{n}=3) . * P<0.05$, Two-side Student's $t$-test. F. After transfection microRNA-141 mimics with plasmid harboring full length CTNNAP1 or microRNA-141 inhibitor, the effect of microRNA-141 on CTNNA1 mRNA level or CTNNAP1 in antagonizing microRNA141 -mediated suppression of $C T N N A 1$ mRNA level was examined by qRT-PCR. The data are presented as the mean $\pm \mathrm{SD}$. ${ }^{*} P<0.05$, Two-side Student's $t$-test. Twenty-four hours after SW480 cells G. and SW620 cells H. were transfected with microRNA-141 mimics, the half-life of $C T N N A P 1$ and $C T N N A 1$ was measured using qRT-PCR. The data represent the Mean \pm SD from three independent experiments. 
Accordingly, the overexpression of CTNNA1 significantly suppressed the colony numbers of the SW480 and SW620 cells compared with the controls (Figure 3D). A similar effect of CTNNAP1 overexpression on colony formation ability was also observed in a parallel with CTNNA1 in SW480 and SW620 cells (Figure 3D). Furthermore, cellcycle progression of transfected SW480 and SW620 cells was measured by using flow cytometry. As shown in Figure 3E and 3F, overexpression of either CTNNAP1 or CTNNA1 caused a cell-cycle arrest, with a significant increase in the proportion of cells in the G0/G1 phase compared with controls in the SW480 cells. Similar results were also observed in SW620 cells.

\section{The CTNNA1 as well as CTNNAP1 inhibited tumorigenesis of CRC in vivo}

We further investigated the effects of overexpression of either CTNNA1 as well as CTNNAP1 on tumor growth in vivo. The CRC cells transfected with pcDNA3.1-
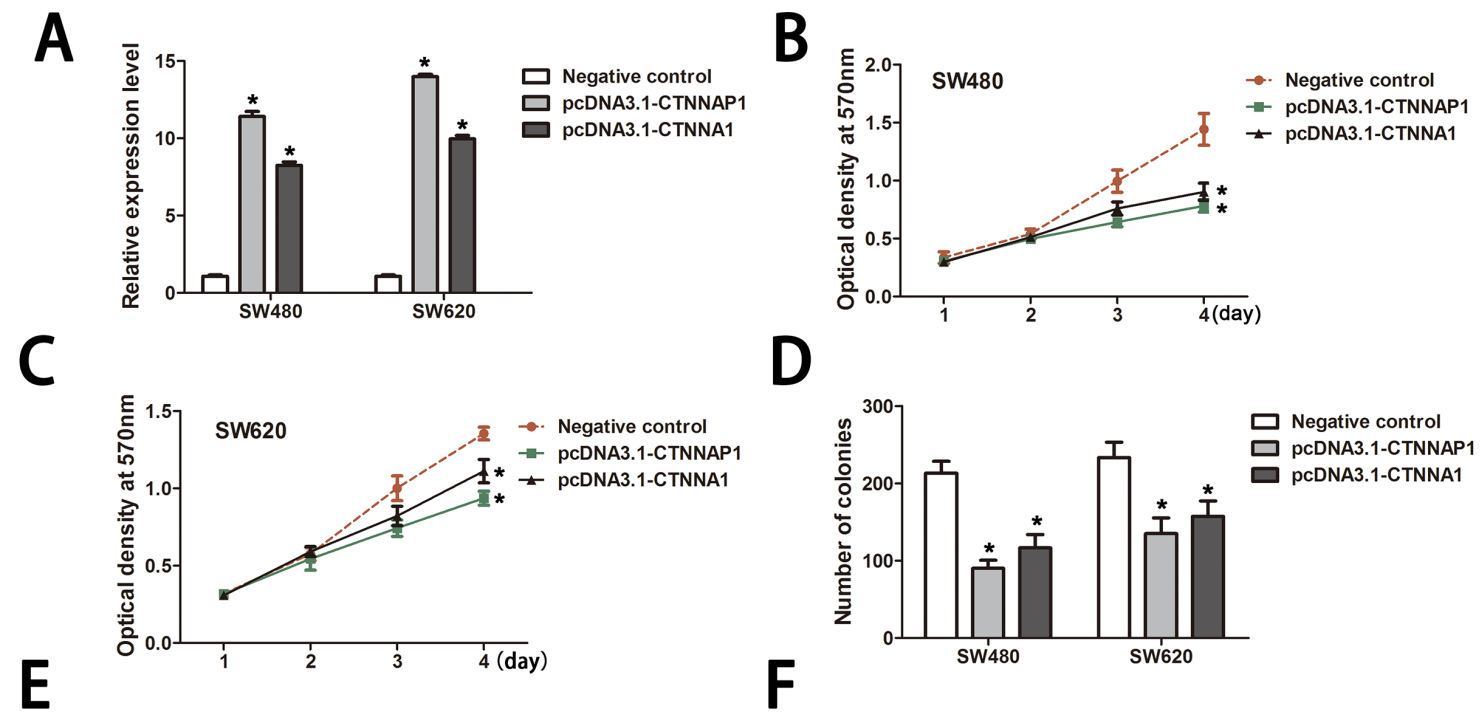

D
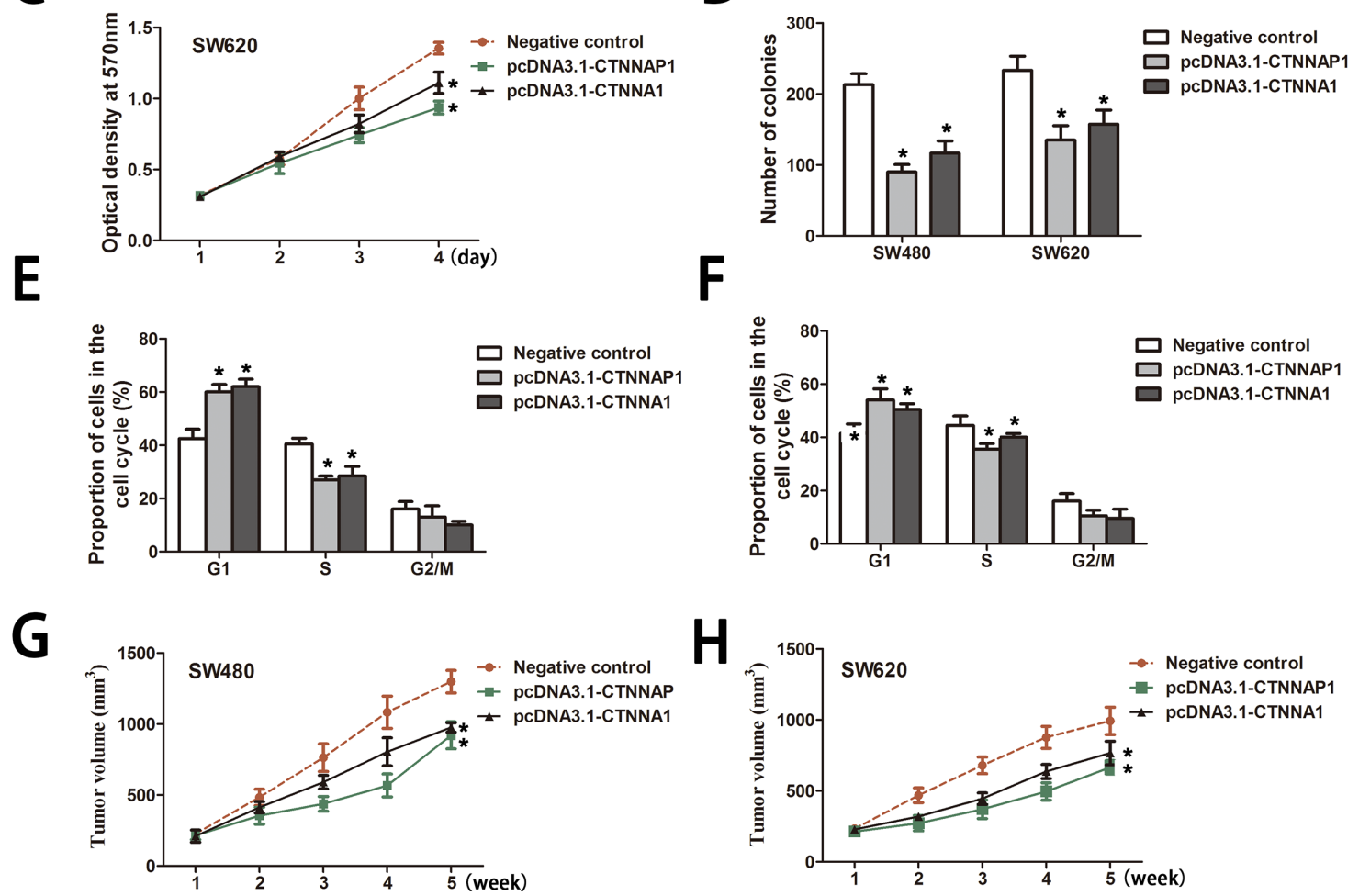

Figure 3: CTNNAP1 and CTNNA1 exert tumor suppressive effects on CRC cells in vitro and in vivo. A. CTNNAP1 and CTNNA1 expression levels were confirmed by qRT-PCR in SW480 cells and SW620 cells. Mean \pm SD are shown $(\mathrm{n}=3)$. $* P<0.05$, Twoside Student's $t$-test. Upregulation of CTNNAP1 and CTNNA1 attenuated CRC cancer cell lines SW480 B. and SW620 C. proliferation at day 4 as determined by CCK8 assay ( $P=0.002$ for $C T N N A P 1$ and $P=0.004$ for $C T N N A 1$ in SW480 cells; $P<0.001$ for $C T N N A P 1$ and $P=0.008$ for $C T N N A 1$ in SW620 cells). ${ }^{*} P<0.05$ compared with the control, Two-sided Student's $t$-test; $\mathrm{n}=6$. D. After two weeks, colony formation of SW480 cells and SW620 cells was significantly suppressed by overexpressing $C T N N A P 1(P<0.001$ in SW480 cells; $P=0.004$ in SW620 cells) or CTNNA1 ( $P=0.002$ in SW480 cells; $P=0.01$ in SW620 cells) when compared to the negative controls. The data are shown (mean $\pm \mathrm{SD}$ ). ${ }^{*} P<0.05$ compared with the control, Two-sided Student's $t$-test; $\mathrm{n}=3$. E. and F. Cell cycle phases of SW480 cells and SW620 cells with $C T N N A P 1$ or $C T N N A 1$ overexpression were determined by Flow cytometry $\left({ }^{*} P<0.05\right.$, Two-sided Student's $t$-test; $\left.\mathrm{n}=3\right)$. G. and H. At week 5, the tumor volumes were dramatically smaller in nude mice injected with CTNNAP1-overexpressed or CTNNA1overexpressed CRC cells compared to nude mice injected with negative control cells. Tumor volumes were determined every three days. The data are presented as the mean $\pm \mathrm{SD} .{ }^{*} P<0.05$, Two-sided Student's $t$-test; $\mathrm{n}=6$. 
CTNNA1 or pcDNA3.1-CTNNAP1 were injected subcutaneously into female nude mice. Five weeks after injection, tumors derived from CTNNA1-overexpression CRC cells were significantly smaller than those derived from empty vector-transfected cells $(976.5 \pm 33.2 \mathrm{~mm} 3$ versus. $1299.0 \pm 79.2 \mathrm{~mm} 3$ for SW480; 766.3 $\pm 83.3 \mathrm{~mm} 3$ versus. 993.3 $\pm 96.3 \mathrm{~mm} 3$ for SW620) (Figure $3 \mathrm{G}$ and $3 \mathrm{H}$ ). In addition, up-regulation of CTNNAP1 in CRC cells induced a similar and smaller tumor size compared with controls $(920.5 \pm 95.5 \mathrm{~mm} 3$ versus. $1299.0 \pm 79.2 \mathrm{~mm} 3$ for SW480; $666.0 \pm 46.9 \mathrm{~mm} 3$ versus. $993.3 \pm 96.3 \mathrm{~mm} 3$ for SW620) (Figure $3 \mathrm{G}$ and $3 \mathrm{H}$ ). These results showed that CTNNAP1 as well as CTNNA1 could obviously inhibit CRC tumorigenesis in vivo. Taken together, the ability of CTNNA1 and CTNNAP1 to suppress cell proliferation and tumor growth indicates that CTNNAP1 and its cognate gene CTNNA1 may potentially play tumor suppressive roles in $\mathrm{CRC}$.

\section{DISCUSSION}

Pseudogenes, as a large component of the human transcriptome, have long been neglected and considered as "junk" DNA [25]. Recently, most of the known pseudogenes have been extensively studied in normal physiology as well as in multiple cancer types [26, 27]. A growing body of evidence have revealed that dysregulation of pseudogenes may regulate the expression of oncogenes or tumor-suppressor genes by acting as modulators of microRNAs $[10,15,28]$. In the current study, we demonstrated for the first time that lower expression of pseudogene CTNNAP1 resulted in CTNNA1 mRNA level suppression by microRNA-141, and conferred a malignant phenotype to colorectal cancer cells lines (Figure 4).

Pseudogene CTNNAP1 is located to human chromosome $5 \mathrm{q} 22$, which was first found from a human genomic phage library. Its cognate gene CTNNA1 plays a central role in cell-cell contact by interacting with cadherin-catenin complex [29]. Clinical observations have intensively revealed the crucial role of CTNNA1 in tumors $[23,30,31]$. However, as a pseudogene for a member of the E-cadherin/catenin complex, it remains unclear whether pseudogene CTNNAP1 has important biological functions. In this study, we found that CTNNAP1 was significantly downregulated in human CRC tissues and patients with lower CTNNAP1 expression levels was significantly correlated with advanced pathological stage. These data imply that pseudogene CTNNAP1 may emerge as a novel player in the development and progression of CRC. To further understand the biological functions of CTNNAP1, we conducted a series of functional experiments to determine the roles of CTNNAP1 in CRC development. Inhibited cell proliferation and tumor growth were observed in CTNNAP1-overexpressed CRC cells. We next put the spotlight on the CTNNAP1 expression influenced tumor-like characteristics, such as
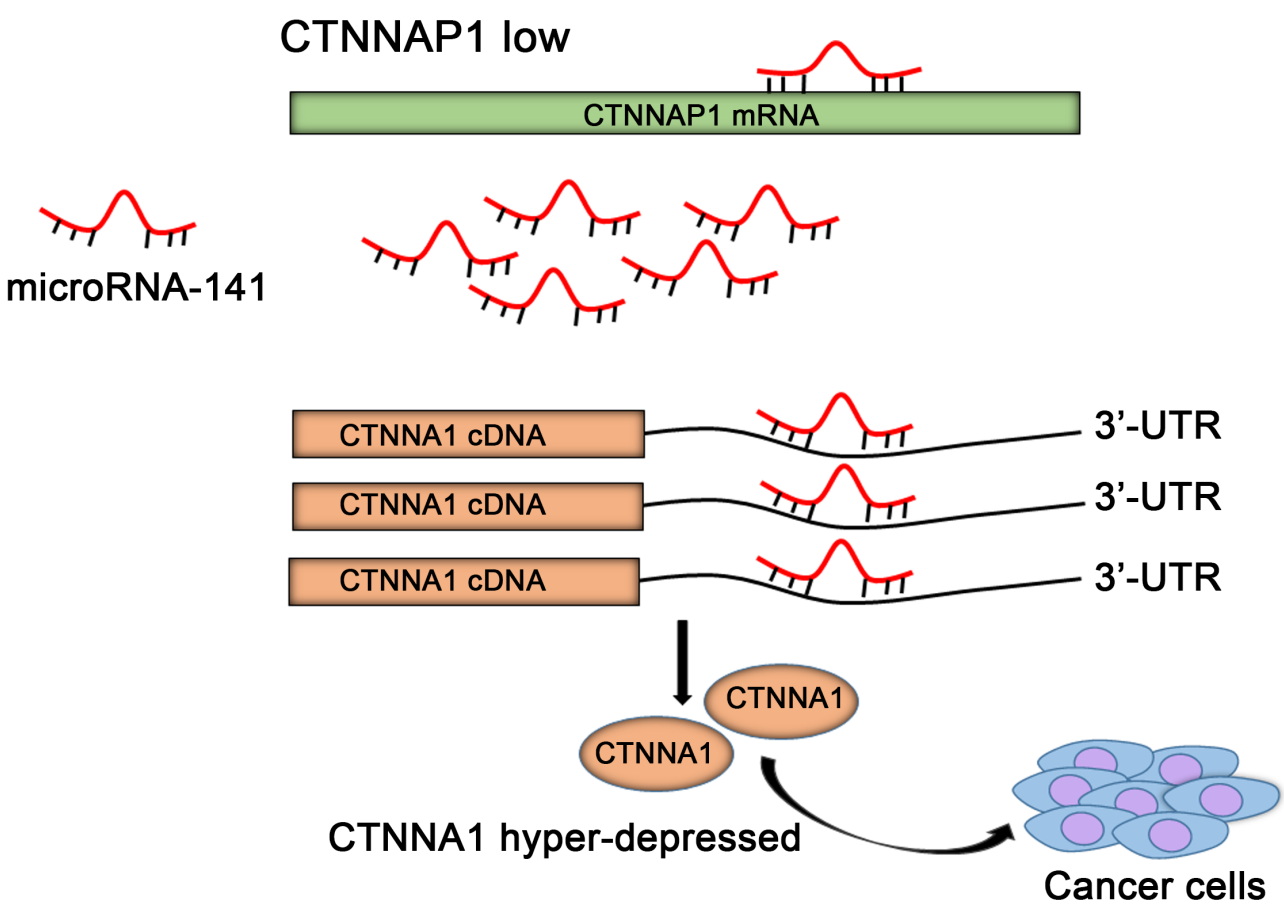

Figure 4: Schematic overview of pseudogene CTNNAP1 regulatory network in CRC pathogenesis. Downregulation of pseudogene CTNNAP1 downregulated its cognate gene CTNNA1 mRNA level via mediating microRNA-141 repression activity, thereby conferred a malignant phenotype to colorectal cancer cells. 
cell cycle progression. Our experiments showed that upregulation of CTNNAP1 in CRC cells led to a significant G1-G0 arrest and a related decrease in S phase. These findings indicate that the proliferation-inhibition effects of CTNNAP1 in CRC probably result from the suppression of the G1-S phase transition.

Further investigating the molecular mechanism through which CTNNAP1 led to the inhibition of CRC cell proliferation and tumor growth in vitro and in vivo. Pseudogene CTNNAP1 exhibits $90 \%$ sequence identity to CTNNA1, which is believed to be important in mediating the linkage between the adhesion molecules E-cadherin and the actin cytoskeleton [32]. The abnormal assembly and expression of E-cadherin-catenin complex would break cell-cell adhesion, resulting in intravasation of primary cancer cells and enhancement of metastases formation [22, 33]. Accumulating evidences have assessed the expression levels of CTNNA1 mRNA in a variety of cancers [34]. In this study, we found that CTNNA1 expression was downregulated in CRC and positively correlated with that of CTNNAP1. Consistently, the functional studies in vitro and in vivo also verified the tumor suppressive roles of CTNNA1 or CTNNAP1 in CRC carcinogenesis. In addition, qRTPCR analysis showed that microRNA-141 expression was inversely correlated with CTNNA1 and CTNNAP1 expression. In recent years, it has been discovered that microRNA-141 can influence DLC1 and SIP1 genes to participate in human diseases, including CRC [35-37]. And in the present study, we showed that CTNNAP1 and CTNNA1 are the major direct target genes of microRNA-141, though the results are not completely consistent with previous studies. Finally, the mechanisms accounting for the correlation expression of CTNNAP1 and CTNNAPl showed that CTNNAPI behaved as a ceRNA to sustain the expression of its parental gene CTNNA1 transcript from being inhibited by microRNA-141. Thus, CTNNAP1 might be a promising candidate target for monitoring CRC.

In summary, the present study has suggested pseudogene CTNNAP1 is a potential tumor suppressor participating in CRC pathogenesis by competing with the parent gene CTNNA1 for microRNA-141. These findings shed a light on the potential of the regulatory network for investigating the underlying mechanisms of $\mathrm{CRC}$ pathogenesis and provided a valuable marker for the monitor of CRC.

\section{MATERIALS AND METHODS}

\section{Tissue collection}

A cohort of 56 CRC patients aged 18-78 years undergoing surgery at the First Affiliated Hospital of Wenzhou Medical University (Wenzhou, China) were enrolled, and written informed was obtained from each subject. The patient who received chemotherapy or radiotherapy prior to surgery were excluded. Clinical characteristics including age, sex, lymph node metastasis, tumor differentiation and TNM stage are shown in Table 1. This study was approved by Ethics Committee of Wenzhou Medical University.

\section{Cell lines}

Human CRC cell lines (SW480 and SW620) were purchased from the American Type Culture Collection (USA). These cell lines were cultured routinely in RPMI Medium 1640 (Invitrogen) supplemented with 10\% fetal bovine serum (Invitrogen, Shanghai, China) and were grown in incubator at $37{ }^{\circ} \mathrm{C}$ with $5 \% \mathrm{CO}_{2}$.

\section{Subcellular fractionation}

For subcellular fractionation experiments, cytosolic and nuclear extracts from CRC cells (SW480 and SW620) were collected using a Nuclear/Cytosol Fractionation kit (Biovision) as previously described [38].

\section{Plasmid constructs and cell transfection}

The cDNAs sequence of CTNNAP1 and CTNNA1 were synthesized and then subcloned into pcDNA3.1 (Invitrogen, Shanghai, China). The microRNA mimics, and microRNA inhibitors were from GenePharma (Shanghai, China). The stable CRC cells with ectopic expression of CTNNAP1 or CTNNA1 were achieved based on previously described method [39]. The empty vector was used as a control. Cells were harvested for quantitative real time RT-PCR (qRT-PCR) after 48h transfection using Lipofectamine 2000 (Invitrogen, Shanghai, China) according to the manufacturer's instructions.

\section{Actinomycin D assay}

To measure half-life of CTNNAP1 and its cognate gene CTNNA1 regulated by microRNA-141. SW480 and SW620 cells were plated in 24-well culture plates. Twenty-four hours after cells were transfected with 40 pmol microRNA-141 mimics (Shanghai GenePharma Co., Ltd.), cells were incubated with Actinomycin D (Sigma) for 2, 4 or $6 \mathrm{~h}$. Actinomycin D was used at a final concentration of $2.5 \mathrm{mg} / \mathrm{ml}$.

\section{QRT-PCR analyses}

Total RNAs of tissues or cultured cells were extracted with TRIzol reagent (Invitrogen, Carlsbad, CA, USA) according to the manufacturer's protocol. The expression of mRNA was evaluated using SYBR Green Assays and microRNA expression was detected using Taqman microRNA Assays (Applied Biosystems) on ABI 7500 system (Applied Biosystems, CA, USA). The 
relative expression was normalized to the expression of glyc-eraldehyde-3-phosphate dehydrogenase (GAPDH) or $U 6$ using the $2^{-\Delta \Delta C t}$. Each sample was analyzed in triplicate.

\section{Bioinformatics prediction and luciferase reporter assay}

We used online software program TargetScan, starbase v2.0 and miRanda databases to predict potential microRNAs that have complementary base pairing with CTNNAP1 and CTNNA1 3'-UTR. The sequence of CTNNA1 3'-UTR containing microRNAs putative target sites or CTNNA1 3'-UTR with point mutations in the microRNA response elements were amplified and then were cloned into psiCHECK-2 vector (promega). The vectors were cotransfected with microRNAs mimics or inhibitors into CRC cells using Lipofectamine 2000 (Invitrogen) for the reporter assay, according to the manufacturer's instructions.

\section{Cell proliferation assays}

CRC cells transfected with pcDNA3.1-CTNNAP1, pcDNA3.1-CTNNA1 or pcDNA3.1 empty vectors were collected and were plated in each well of a 96-well plate. Cell viability was measured every $24 \mathrm{~h}$ by the Cell Counting Kit-8 (CCK-8) kit. For the colony formation assay, approximately $300 \mathrm{CRC}$ cells transfected with pcDNA3.1-CTNNAP1, pcDNA3.1-CTNNA1 or pcDNA3.1 empty vectors were plated into per well for six-well plates for 2 weeks incubation. The colonies were counted after fixing with methanol and staining with crystal violet (Sigma, USA) according to the manufacturer's instructions.

\section{Flow-cytometric analysis of cell cycle}

These CRC cells transfected with pcDNA3.1CTNNAP1, pcDNA3.1-CTNNA1 or pcDNA3.1 empty vectors with overexpressed CTNNAPI or CTNNA1 as described above were plated in six-well plates. After cultivation for $48 \mathrm{~h}$, the cells were harvested and subjected to analyze for cell cycle by a flow cytometer (FACSCalibur, BD Biosciences) according to the manufacturer's instructions. Each experiments was repeated three times independently.

\section{Xenograft studies}

All female athymic BALB/c mice (5-week-old) were purchased from the Shanghai Experimental Animal Center of the Chinese Academy of Sciences and randomly were divided into control or experimental group. The animal study protocol was approved by the Animal Experimentation Ethics Committee of the Third Affiliated Hospital of Harbin Medical University. For a CRC mouse model system, $5 \times 10^{6} \mathrm{CRC}$ cell lines with overexpressed CTNNAP1 or CTNNA1 were injected subcutaneously in the posterior flank of BALB/c nude mice (6 mice per group). Tumor volumes were calculated every 3 days by measuring the length and width with calipers (Tumor volumes $=0.5 \times$ length $\times$ width $^{2}$ ).

\section{Statistical analysis}

An unpaired two-tailed student's t-test and oneway analysis of variance (ANOVA) test were used to evaluate the significance of the differences. The expression relationship between CTNNAP1, CTNNA1 and microRNAs in tissues was determined via linear regression model. Statistical analysis was performed using SPSS software (SPSS, Inc., Chicago, IL, USA). $P$ values $<0.05$ was defined as statistically significant.

\section{ACKNOWLEDGMENTS}

This work was supported by a grant from Natural Science Foundation of Zhejiang Province of China (LY16H160048), Wenzhou Science and Technology Bureau Project (Y20150050 and Y20150029).

\section{CONFLICTS OF INTEREST}

The authors declare no conflicts of interest.

\section{REFERENCES}

1. Jemal A, Siegel R, Ward E, Hao Y, Xu J, Thun MJ. Cancer statistics, 2009. CA Cancer J Clin. 2009; 59:225-249.

2. Huxley RR, Ansary-Moghaddam A, Clifton P, Czernichow S, Parr CL, Woodward M. The impact of dietary and lifestyle risk factors on risk of colorectal cancer: a quantitative overview of the epidemiological evidence. International journal of cancer Journal international du cancer. 2009; 125:171-180.

3. Tsong WH, Koh WP, Yuan JM, Wang R, Sun CL, Yu MC. Cigarettes and alcohol in relation to colorectal cancer: the Singapore Chinese Health Study. British journal of cancer. 2007; 96:821-827.

4. Guffanti A, Iacono M, Pelucchi P, Kim N, Solda G, Croft LJ, Taft RJ, Rizzi E, Askarian-Amiri M, Bonnal RJ, Callari M, Mignone F, Pesole G, et al. A transcriptional sketch of a primary human breast cancer by 454 deep sequencing. BMC genomics. 2009; 10:163.

5. Matouk IJ, Abbasi I, Hochberg A, Galun E, Dweik H, Akkawi M. Highly upregulated in liver cancer noncoding RNA is overexpressed in hepatic colorectal metastasis. European journal of gastroenterology \& hepatology. 2009; 21:688-692. 
6. Prensner JR, Iyer MK, Balbin OA, Dhanasekaran SM, Cao Q, Brenner JC, Laxman B, Asangani IA, Grasso CS, Kominsky HD, Cao X, Jing X, Wang X, et al. Transcriptome sequencing across a prostate cancer cohort identifies PCAT-1, an unannotated lincRNA implicated in disease progression. Nature biotechnology. 2011; 29:742-749.

7. Li N, Zheng J, Li H, Deng J, Hu M, Wu H, Li W, Li F, Lan $\mathrm{X}$, Lu J, Zhou Y. Identification of chimeric TSNAX-DISC1 resulting from intergenic splicing in endometrial carcinoma through high-throughput RNA sequencing. Carcinogenesis. 2014; 35:2687-2697.

8. Khelifi A, Duret L, Mouchiroud D. HOPPSIGEN: a database of human and mouse processed pseudogenes. Nucleic acids research. 2005; 33:D59-66.

9. Mighell AJ, Smith NR, Robinson PA, Markham AF. Vertebrate pseudogenes. FEBS letters. 2000; 468:109-114.

10. Wang L, Guo ZY, Zhang R, Xin B, Chen R, Zhao J, Wang T, Wen WH, Jia LT, Yao LB, Yang AG. Pseudogene OCT4-pg4 functions as a natural micro RNA sponge to regulate OCT4 expression by competing for miR-145 in hepatocellular carcinoma. Carcinogenesis. 2013; 34:1773-1781.

11. Han L, Yuan Y, Zheng S, Yang Y, Li J, Edgerton ME, Diao L, Xu Y, Verhaak RG, Liang H. The Pan-Cancer analysis of pseudogene expression reveals biologically and clinically relevant tumour subtypes. Nature communications. 2014; 5:3963.

12. Scarola M, Comisso E, Pascolo R, Chiaradia R, Maria Marion R, Schneider C, Blasco MA, Schoeftner S, Benetti R. Epigenetic silencing of Oct4 by a complex containing SUV39H1 and Oct4 pseudogene lncRNA. Nature communications. 2015; 6:7631.

13. Welch JD, Baran-Gale J, Perou CM, Sethupathy P, Prins JF. Pseudogenes transcribed in breast invasive carcinoma show subtype-specific expression and ceRNA potential. BMC genomics. 2015; 16:113.

14. Salmena L, Poliseno L, Tay Y, Kats L, Pandolfi PP. A ceRNA hypothesis: the Rosetta Stone of a hidden RNA language? Cell. 2011; 146:353-358.

15. Yu G, Yao W, Gumireddy K, Li A, Wang J, Xiao W, Chen K, Xiao H, Li H, Tang K, Ye Z, Huang Q, Xu H. Pseudogene PTENP1 functions as a competing endogenous RNA to suppress clear-cell renal cell carcinoma progression. Molecular cancer therapeutics. 2014; 13:3086-3097.

16. Yang W, Du WW, Li X, Yee AJ, Yang BB. Foxo3 activity promoted by non-coding effects of circular RNA and Foxo3 pseudogene in the inhibition of tumor growth and angiogenesis. Oncogene. 2015.

17. Peng H, Ishida M, Li L, Saito A, Kamiya A, Hamilton JP, Fu R, Olaru AV, An F, Popescu I, Iacob R, Dima S, Alexandrescu ST, et al. Pseudogene INTS6P1 regulates its cognate gene INTS6 through competitive binding of miR-17-5p in hepatocellular carcinoma. Oncotarget. 2015; 6:5666-5677. doi: 10.18632/oncotarget.3290.
18. Poliseno L, Salmena L, Zhang J, Carver B, Haveman WJ, Pandolfi PP. A coding-independent function of gene and pseudogene mRNAs regulates tumour biology. Nature. 2010; 465:1033-1038.

19. Wu PP, Zhu HY, Sun XF, Chen LX, Zhou Q, Chen J. MicroRNA-141 regulates the tumour suppressor DLC1 in colorectal cancer. Neoplasma. 2015; 62:705-712.

20. Nollet F, van Hengel J, Berx G, Molemans F, van Roy F. Isolation and characterization of a human pseudogene (CTNNAP1) for alpha E-catenin (CTNNA1): assignment of the pseudogene to $5 \mathrm{q} 22$ and the alpha E-catenin gene to 5q31. Genomics. 1995; 26:410-413.

21. Lee B, Yoon K, Lee S, Kang JM, Kim J, Shim SH, Kim HM, Song S, Naka K, Kim AK, Yang HK, Kim SJ. Homozygous deletions at 3p22, 5p14, 6q15, and 9p21 result in aberrant expression of tumor suppressor genes in gastric cancer. Genes, chromosomes \& cancer. 2015; 54:142-155.

22. Vite A, Li J, Radice GL. New functions for alpha-catenins in health and disease: from cancer to heart regeneration. Cell and tissue research. 2015; 360:773-783.

23. Piao HL, Yuan Y, Wang M, Sun Y, Liang H, Ma L. alphacatenin acts as a tumour suppressor in E-cadherin-negative basal-like breast cancer by inhibiting NF-kappaB signalling. Nature cell biology. 2014; 16:245-254.

24. Zhou YN, Xu CP, Chen Y, Han B, Yang SM, Fang DC. Alpha-catenin expression is decreased in patients with gastric carcinoma. World journal of gastroenterology. 2005; 11:3468-3472.

25. Li WH, Gojobori T, Nei M. Pseudogenes as a paradigm of neutral evolution. Nature. 1981; 292:237-239.

26. Pink RC, Wicks K, Caley DP, Punch EK, Jacobs L, Carter DR. Pseudogenes: pseudo-functional or key regulators in health and disease? Rna. 2011; 17:792-798.

27. Poliseno L. Pseudogenes: newly discovered players in human cancer. Science signaling. 2012; 5:re5.

28. Rutnam ZJ, Du WW, Yang W, Yang X, Yang BB. The pseudogene TUSC2P promotes TUSC2 function by binding multiple microRNAs. Nature communications. 2014; 5:2914.

29. Pokutta S, Drees F, Yamada S, Nelson WJ, Weis WI. Biochemical and structural analysis of alpha-catenin in cell-cell contacts. Biochemical Society transactions. 2008; 36:141-147.

30. Sygut A, Przybylowska K, Ferenc T, Dziki L, Spychalski M, Mik M, Dziki A. Genetic variations of the CTNNA1 and the CTNNB1 genes in sporadic colorectal cancer in Polish population. Polski przeglad chirurgiczny. 2012; 84:560-564.

31. Majewski IJ, Kluijt I, Cats A, Scerri TS, de Jong D, Kluin RJ, Hansford S, Hogervorst FB, Bosma AJ, Hofland I, Winter M, Huntsman D, Jonkers J, Bahlo M, Bernards R. An alpha-E-catenin (CTNNA1) mutation in hereditary diffuse gastric cancer. The Journal of pathology. 2013; 229:621-629. 
32. Chen CS, Hong S, Indra I, Sergeeva AP, Troyanovsky RB, Shapiro L, Honig B, Troyanovsky SM. alpha-Cateninmediated cadherin clustering couples cadherin and actin dynamics. The Journal of cell biology. 2015; 210:647-661.

33. Hinck L, Nathke IS, Papkoff J, Nelson WJ. Dynamics of cadherin/catenin complex formation: novel protein interactions and pathways of complex assembly. The Journal of cell biology. 1994; 125:1327-1340.

34. Pierceall WE, Woodard AS, Morrow JS, Rimm D, Fearon ER. Frequent alterations in E-cadherin and alpha- and beta-catenin expression in human breast cancer cell lines. Oncogene. 1995; 11:1319-1326.

35. Hu M, Xia M, Chen X, Lin Z, Xu Y, Ma Y, Su L. MicroRNA-141 regulates Smad interacting protein 1 (SIP1) and inhibits migration and invasion of colorectal cancer cells. Digestive diseases and sciences. 2010; 55:2365-2372.

36. Banaudha K, Kaliszewski M, Korolnek T, Florea L, Yeung ML, Jeang KT, Kumar A. MicroRNA silencing of tumor suppressor DLC-1 promotes efficient hepatitis C virus replication in primary human hepatocytes. Hepatology. 2011; 53:53-61.

37. Gregory PA, Bert AG, Paterson EL, Barry SC, Tsykin A, Farshid G, Vadas MA, Khew-Goodall Y, Goodall GJ. The miR-200 family and miR-205 regulate epithelial to mesenchymal transition by targeting ZEB1 and SIP1. Nature cell biology. 2008; 10:593-601.

38. Li W, Zheng J, Deng J, You Y, Wu H, Li N, Lu J, Zhou Y. Increased levels of the long intergenic non-protein coding RNA POU3F3 promote DNA methylation in esophageal squamous cell carcinoma cells. Gastroenterology. 2014; 146:1714-1726 e1715.

39. Zheng J, Deng J, Xiao M, Yang L, Zhang L, You Y, Hu M, Li N, Wu H, Li W, Lu J, Zhou Y. A sequence polymorphism in miR-608 predicts recurrence after radiotherapy for nasopharyngeal carcinoma. Cancer research. 2013; 73:5151-5162. 